\title{
EDGEWORTH EXPANSIONS FOR SPECTRAL MEAN ESTIMATES WITH APPLICATIONS TO WHITTLE ESTIMATES
}

\author{
Daniel Janas \\ Institut für Angewandte Mathematik \\ Universität Heidelberg \\ W-6900 Heidelberg \\ Germany
}

\begin{abstract}
We prove that the distributions of spectral mean estimates from linear processes admit Edgeworth expansions. As a consequence, Edgeworth expansions are valid for Whittle estimates.
\end{abstract}

\section{Introduction}

We consider a real-valued stationary time series $\left\{X_{t}\right\}_{t \in \mathbf{Z}}$ with $\mathbf{E X} 1=0$ and spectral density f. Let us denote by

$$
A(\phi, f) \equiv\left(\int_{0}^{\pi} \phi^{(1)}(\alpha) f(\alpha) d \alpha, \ldots, \int_{0}^{\pi} \phi^{(d)}(\alpha) f(\alpha) d \alpha\right)^{\prime}\left(\equiv \int \phi f\right)
$$

the spectral mean, where $\phi^{(\mathrm{r})}$ are functions of bounded variation for $\mathrm{r}=1, \ldots, \mathrm{d}$. The canonical estimate of $\mathrm{A}(\phi, f)$ is

$$
\mathrm{A}\left(\phi, \mathrm{I}_{\mathrm{T}}\right) \equiv\left(\int_{0}^{\pi} \phi^{(1)}(\alpha) \mathrm{I}_{\mathrm{T}}(\alpha) \mathrm{d} \alpha, \ldots, \int_{0}^{\pi} \phi^{(\mathrm{d})}(\alpha) \mathrm{I}_{\mathrm{T}}(\alpha) \mathrm{d} \alpha\right)^{\prime} \quad\left(\equiv \int \phi \mathrm{I}_{\mathrm{T}}\right)
$$

where $\mathrm{I}_{\mathrm{T}}$ is the tapered periodogram, i.e.

$$
\mathrm{I}_{\mathrm{T}}(\alpha) \equiv\left(2 \pi \mathrm{H}_{2, \mathrm{~T}}\right)^{-1}\left|\sum_{\mathrm{t}=1}^{\mathrm{T}} \mathrm{h}_{\mathrm{t}} \mathrm{X}_{\mathrm{t}} \exp (-\mathrm{i} \alpha \mathrm{t})\right|^{2}
$$

(cf. Dahlhaus (1983)).

By a different choice for the function $\phi$ we get estimates for the autovariances at different lags, the spectral distribution function and the spectral density function at a

Key words and phrases: Cramér's condition, Edgeworth expansion, linear process, spectral mean estimates, Whittle estimates. 
finite number of points as well as quantities that are needed to compute the Whittle estimates.

If the underlying process $\left\{\mathrm{X}_{\mathrm{t}}\right\}$ is Gaussian, Edgeworth expansions of the statistic in (1.2) have been given for $d=1$ and special $\phi$ 's in the nontapered case by several authors: Bentkus (1982) proves an expansion for kernel spectral density estimates and Taniguchi (1991) shows the validity of Edgeworth expansions of generalized maximum likelihood estimators for Gaussian ARMA-processes. Bose (1988) drops the assumption of Gaussianity. He gives higher order approximations for a vector of autocovariances from a linear process.

In this paper we establish Edgeworth expansions for the distribution of the statistic given in (1.2) when the process is linear. The expansions are valid for $\phi$ 's whose Fourier coefficients decrease exponentially. The data are allowed to be tapered. As an application of this result we show that the distributions of the Whittle estimates admit Edgeworth expansions.

The paper is organized as follows: In section 2 we give the main results that include a basic theorem for Edgeworth expansions for sums of dependent random vectors by Götze and Hipp (1983). The application of these results to the Whittle estimates is found in section 3. In order to make the paper more convenient for the reader we have transferred all proofs to section 4.

\section{Main results}

First we gather the assumptions needed in this paper:

(A1) $\quad\left\{\mathrm{X}_{\mathrm{t}}\right\}_{\mathrm{t} \in \mathbf{Z}}$ is a real-valued linear process such that $\mathrm{X}_{\mathrm{t}}=\sum_{\mathrm{u} \in \mathbf{Z}} \mathrm{a}_{\mathrm{u}} \varepsilon_{\mathrm{t}-\mathrm{u}}$, where $\varepsilon_{\mathrm{t}}$ are i.i.d. random variables satisfying $\mathbf{E} \varepsilon_{1}=0, \mathbf{E} \varepsilon_{1}^{2}=1, \mathbf{E} \varepsilon_{1}^{3}=0$, E $\varepsilon_{1}^{2(s+1)}<\infty$ for some fixed $\mathrm{s} \geq 3$.

(A2) $\quad\left(\varepsilon_{1}, \varepsilon_{1}^{2}\right)$ fulfills Cramér's condition, i.e. $\exists \delta>0, \mathrm{~d}>0 \quad \forall\|\mathrm{t}\|>\mathrm{d}$ $\left|\mathbf{E} \exp \left(\mathrm{it}^{\prime}\left(\varepsilon_{1}, \varepsilon_{1}^{2}\right)^{\prime}\right)\right| \leq 1-\delta$.

(A3) The filter coefficients $a_{u}$ and the Fourier coefficients $\hat{\phi}(u)$ of $\phi$ decrease exponentially, i.e.

$$
\exists 0<\rho<1 \quad \forall \text { large } u \quad\left|a_{u}\right|<\rho^{|u|}, \quad\|\hat{\phi}(u)\|<\rho^{|u|} .
$$


(A4) The data taper $\mathrm{h}: \mathbf{R} \rightarrow[0,1]$ is twice continuously differentiable,

$$
h(x)=0 \quad \text { for } x \notin(0,1) \text { and } \quad H_{2} \equiv \int_{0}^{1} h^{2}(x) d x>0 \text {. }
$$

(A5) $\quad \sum=\lim _{\mathrm{T} \rightarrow \infty} \mathrm{D}\left(\sqrt{\mathrm{T}} \int_{\phi \mathrm{I}_{\mathrm{T}}}\right)$ is positive definite, where $\mathrm{D}$ denotes the dispersion matrix.

\section{Remark 2.1.}

(1) The assumption that the third moment of $\varepsilon_{1}$ is zero can be dropped. It is only made for convenience.

(2) The minimum assumption we need is $\mathbf{E} \varepsilon_{1}^{8}<\infty$. The reason is that the statistics considered involve quadratic functions of $\varepsilon_{\mathrm{t}}$ and Edgeworth expansions for sums of dependent random vectors require the $(s+1)$-th moment of $\varepsilon_{t}^{2}$ with s at least three.

In order to derive our main results we take the help of the following results of Götze and Hipp (1983) (henceforth referred to as GH).

Let $\left\{\mathrm{Z}_{\mathrm{T}, \mathrm{t}}\right\}_{\mathrm{t}=1, \ldots, \mathrm{T}}$ be a triangular array of $\mathrm{d}$-dimensional, real-valued random vectors on an abstract measure space $(\Omega, \mathcal{A}, \mathrm{P})$ with $\mathbf{E} \mathrm{Z}_{\mathrm{T}, \mathrm{t}}=0 \quad \forall \mathrm{t}$ and

$$
\mathrm{S}_{\mathrm{T}}=\mathrm{c}_{\mathrm{T}}^{-1 / 2} \sum_{\mathrm{t}=1}^{\mathrm{T}} \mathrm{Z}_{\mathrm{T}, \mathrm{t}}
$$

where $\mathrm{c}_{\mathrm{T}}$ is a norming constant of order $\mathrm{T}$ to be specified. The function $\Psi_{\mathrm{T}, \mathrm{s}}$ represents the first $(s-1)$ terms of the Edgeworth expansion of the distribution of $S_{T}$ whenever such an expansion is valid. For any random vector $Z, D(Z)$ denotes the dispersion matrix of $Z$. Let $\varphi_{\Sigma}$ be the normal density with mean zero and dispersion matrix $\sum$, and $\Phi_{\Sigma}$ the corresponding distribution function. c stands for a generic constant. Let $\mathrm{f}: \mathbf{R}^{\mathrm{d}} \rightarrow \mathbf{R}$ be a measurable function with $M_{r}(f) \equiv \sup _{x}(1+\|x\|)^{-r}|f(x)|<\infty$. Define the average modulus of oscillation of $f$ with respect to a finite measure $P$ by $\bar{\omega}(f, \varepsilon, P) \equiv \int_{\|y-x\| \leq \varepsilon}|f(y)-f(x)| d P(x)$.

Let $\mathcal{D}_{\mathrm{j}}$ be $\sigma$-fields on $(\Omega, \mathcal{A}, \mathrm{P})\left(\right.$ write $\left.\sigma\left(\bigcup_{\mathrm{j}=\mathrm{a}}^{\mathrm{b}} \mathcal{D}_{\mathrm{j}}\right) \equiv \mathcal{D}_{\mathrm{a}}^{\mathrm{b}}\right)$ and $0<\rho<1$ such that 
C(1) $\quad \mathbf{E} \mathrm{Z}_{\mathrm{T}, \mathrm{t}}=0 \quad \forall \mathrm{t}$.

$\mathbf{C ( 2 )} \quad \mathbf{E}\left\|\mathrm{Z}_{\mathrm{T}, \mathrm{t}}\right\|^{\mathrm{s}+1} \leq \beta_{\mathrm{s}+1}<\infty \quad \forall \mathrm{t}$ for some $\mathrm{s} \geq 3$.

$\mathbf{C ( 3 )} \quad \exists \mathrm{Y}_{\mathrm{T}, \mathrm{t}, \mathrm{m}} \in \mathcal{D}_{\mathrm{t}-\mathrm{m}}^{\mathrm{t}+\mathrm{m}} \quad$ with $\quad \mathbf{E}\left\|\mathrm{Z}_{\mathrm{T}, \mathrm{t}}-\mathrm{Y}_{\mathrm{T}, \mathrm{t}, \mathrm{m}}\right\| \leq \rho^{\mathrm{m}}$.

C(4) $\quad \forall \mathrm{A} \in \mathcal{D}_{-\infty}^{\mathrm{t}}, \mathrm{B} \in \mathcal{D}_{\mathrm{t}+\mathrm{m}}^{\infty} \quad|\mathrm{P}(\mathrm{A} \cap \mathrm{B})-\mathrm{P}(\mathrm{A}) \mathrm{P}(\mathrm{B})| \leq \rho^{\mathrm{m}}$.

C(5) $\quad \exists \varepsilon, \eta, \rho>0 \quad \forall\|\theta\| \geq \varepsilon \quad \forall \rho^{-1}<\mathrm{m}<\mathrm{T}$

$\#\left\{t \in\{1, \ldots, \mathrm{T}\}: \mathbf{E}\left|\mathbf{E} \exp \left(\mathrm{i} \theta^{\prime}\left(\mathrm{Z}_{\mathrm{T}, \mathrm{t}-\mathrm{m}}+\ldots+\mathrm{Z}_{\mathrm{T}, \mathrm{t}+\mathrm{m}}\right) \mid \mathcal{D}_{\mathrm{j}}: \mathrm{j} \neq \mathrm{t}\right)\right| \leq 1-\eta\right\} \geq \rho \mathrm{T}$

$\mathbf{C ( 6 )} \quad \forall \mathrm{A} \in \mathcal{D}_{\mathrm{t}-\mathrm{p}}^{\mathrm{t}+\mathrm{p}} \quad \forall \mathrm{t}, \mathrm{p}, \mathrm{m} \quad \mathbf{E}\left|\mathrm{P}\left(\mathrm{A} \mid \mathcal{D}_{\mathrm{j}}: \mathrm{j} \neq \mathrm{t}\right)-\mathrm{P}\left(\mathrm{A}\left|\mathcal{D}_{\mathrm{j}}: 0<\right| \mathrm{j}-\mathrm{t} \mid \leq \mathrm{m}+\mathrm{p}\right)\right| \leq \rho^{\mathrm{m}}$

C(7) $\quad \lim _{\mathrm{T} \rightarrow \infty} \mathrm{D}\left(\mathrm{S}_{\mathrm{T}}\right)=\sum$ exists and is positive definite.

\section{Remark.}

The Cramér type condition $\mathrm{C}(5)$ is a weaker assumption than the condition (2.5) in GH. Nevertheless, it suffices for the results of $\mathrm{GH}$ to hold as is pointed out by remark (3.44) in $\mathrm{GH}$. The weaker condition $\mathrm{C}(5)$ means that Cramér's condition is fulfilled for a sufficiently large number of t's. Whereas condition (2.5) cannot be fulfilled in the situations we will discuss, by some effort it is possible to verify $\mathrm{C}(5)$.

Let $\mathrm{s}_{0}$ be $\mathrm{s}$ or $(\mathrm{s}-1)$ according to $\mathrm{s}$ is even or odd.

\section{Theorem 2.1.}

Assume that $\mathrm{C}(1)-\mathrm{C}(7)$ hold. Then there exists a positive constant $\delta$ not depending on $\mathrm{f}$ and $\mathrm{M}_{\mathrm{s}_{0}}(\mathrm{f})$, and for arbitrary $\mathrm{\kappa}>0$ there exists a positive constant $\mathrm{c}$ depending on $\mathrm{M}_{\mathrm{s}_{0}}(\mathrm{f})$ but not on $f$ such that

$$
\left|\mathbf{E ~} \mathrm{f}\left(\mathrm{S}_{\mathrm{T}}\right)-\int \mathrm{fd} \Psi_{\mathrm{T}, \mathrm{s}}\right| \leq \mathrm{c} \bar{\omega}\left(\mathrm{f}, \mathrm{T}^{-\kappa}, \Phi_{\Sigma}\right)+\mathrm{o}\left(\mathrm{T}^{-(\mathrm{s}-2+\delta) / 2)}\right.
$$

The term $\mathrm{o}(\cdot)$ depends on $f$ through $\mathrm{M}_{\mathrm{s}_{0}}(\mathrm{f})$ only. 


\section{Corollary 2.2.}

Assume $\mathrm{C}(1)-\mathrm{C}(7)$. Then the following approximation holds uniformly over convex measurable $\mathrm{C} \subseteq \mathbf{R}^{\mathrm{d}}$ :

$$
\mathrm{P}\left(\mathrm{S}_{\mathrm{T}} \in \mathrm{C}\right)=\Psi_{\mathrm{T}, \mathrm{s}}(\mathrm{C})+\mathrm{o}\left(\mathrm{T}^{-(\mathrm{s}-2) / 2}\right)
$$

To apply GH to the distribution of a spectral mean estimate first of all we have to find a representation of the statistic of interest in (1.2) as a sum of appropiate random vectors.

Parseval's identity implies

$$
\int_{0}^{\pi} \phi^{(j)}(\alpha) I_{T}(\alpha) d \alpha=\frac{1}{2 \pi} \sum_{|r| \leq T} \hat{\phi}^{(j)}(r) c_{T}(r)
$$

where $\hat{\phi}^{(\mathrm{j})}(\mathrm{r}) \equiv \int_{0}^{\pi} \phi^{(\mathrm{j})}(\alpha) \cos (\alpha \mathrm{r}) \mathrm{d} \alpha$ are the Fourier coefficients of $\phi^{(\mathrm{j})}$ and $\mathrm{c}_{\mathrm{T}}(\mathrm{r}) \equiv \mathrm{H}_{2, \mathrm{~T}}^{-1} \sum_{1 \leq \mathrm{t}, \mathrm{r} \leq \mathrm{T}} \mathrm{h}_{\mathrm{t}} \mathrm{X}_{\mathrm{t}} \mathrm{h}_{\mathrm{t}+\mathrm{r}} \mathrm{X}_{\mathrm{t}+\mathrm{r}}$ is the tapered autocovariance estimate of $\left\{\mathrm{X}_{\mathrm{t}}\right\}$.

If $\phi^{(j)}$ are even, real-valued functions, we get $\hat{\phi}^{(j)}(r)=\widehat{\phi}^{(j)}(-r)$ for $r \in \mathbf{Z}$ (otherwise consider the even extension of $\phi^{(j)}$ ).

Equally, we have $c_{T}(r)=c_{T}(-r)$ for $r \in Z$.

With $\widehat{\psi}^{(\mathrm{j})}(0) \equiv \widehat{\phi}^{(\mathrm{j})}(0)$ and $\widehat{\psi}^{(\mathrm{j})}(\mathrm{r}) \equiv 2 \widehat{\phi}^{(\mathrm{j})}(\mathrm{r}) \quad$ for $\mathrm{r} \neq 0$ we obtain further

$$
\begin{aligned}
\frac{1}{2 \pi} \sum_{\mathrm{r}=0}^{\mathrm{T}} \widehat{\psi}^{(\mathrm{j})}(\mathrm{r}) \mathrm{c}_{\mathrm{T}}(\mathrm{r}) & =\left(2 \pi \mathrm{H}_{2, \mathrm{~T}}\right)^{-1} \sum_{\mathrm{r}=0}^{\mathrm{T}} \widehat{\psi}^{(\mathrm{j})}(\mathrm{r}) \sum_{\mathrm{t}=1}^{\mathrm{T}} \mathrm{h}_{\mathrm{t}} \mathrm{X}_{\mathrm{t}} \mathrm{h}_{\mathrm{t}+\mathrm{r}} \mathrm{X}_{\mathrm{t}+\mathrm{r}} \\
& =\left(2 \pi \mathrm{H}_{2, \mathrm{~T}}\right)^{-1} \sum_{\mathrm{t}=1}^{\mathrm{T}} \sum_{\mathrm{r}=0}^{\mathrm{T}} \widehat{\psi}^{(\mathrm{j})}(\mathrm{r}) \mathrm{h}_{\mathrm{t}} \mathrm{h}_{\mathrm{t}+\mathrm{r}} \mathrm{X}_{\mathrm{t}} \mathrm{X}_{\mathrm{t}+\mathrm{r}},
\end{aligned}
$$

since $h(r)=0$ for $|r|>1$. Let

$$
\mathrm{U}_{\mathrm{T}, \mathrm{t}} \equiv\left(\sum_{\mathrm{r}=0}^{\mathrm{T}} \widehat{\psi}^{(\mathrm{j})}(\mathrm{r}) \mathrm{h}_{\mathrm{t}} \mathrm{h}_{\mathrm{t}+\mathrm{r}} \mathrm{X}_{\mathrm{t}} \mathrm{X}_{\mathrm{t}+\mathrm{r}}\right)_{\mathrm{j}=1, \ldots, \mathrm{d}}^{\prime}\left(\text { write } \sum_{\mathrm{r}=0}^{\mathrm{T}} \widehat{\psi}(\mathrm{r}) \mathrm{h}_{\mathrm{t}} \mathrm{h}_{\mathrm{t}+\mathrm{r}} \mathrm{X}_{\mathrm{t}} \mathrm{X}_{\mathrm{t}+\mathrm{r}}\right),
$$




$$
\mathrm{Z}_{\mathrm{T}, \mathrm{t}} \equiv \mathrm{U}_{\mathrm{T}, \mathrm{t}}-\mathbf{E} \mathrm{U}_{\mathrm{T}, \mathrm{t}}
$$

and

$$
\mathrm{c}_{\mathrm{T}}^{-1 / 2} \equiv \mathrm{T}^{1 / 2} /\left(2 \pi \mathrm{H}_{2, \mathrm{~T}}\right)
$$

Then the standardized version of (1.2), i.e.

$$
\sqrt{\mathrm{T}}\left(\int_{0}^{\pi} \phi(\alpha) \mathrm{I}_{\mathrm{T}}(\alpha) \mathrm{d} \alpha-\mathbf{E} \int_{0}^{\pi} \phi(\alpha) \mathrm{I}_{\mathrm{T}}(\alpha) \mathrm{d} \alpha\right)
$$

may be rewritten as

$$
\mathrm{S}_{\mathrm{T}} \equiv \mathrm{c}_{\mathrm{T}}^{-1 / 2} \sum_{\mathrm{t}=1}^{\mathrm{T}} \mathrm{Z}_{\mathrm{T}, \mathrm{t}}
$$

We now state our main theorem.

\section{Theorem 2.3.}

Under conditions (A1) - (A5) theorem 2.1 and corollary 2.2 hold for $\mathrm{S}_{\mathrm{T}}$ defined in (2.4).

\section{Remark 2.4.}

(1) As in Theorem 2.10 of Götze and Hipp (1983) we can replace the Cramér condition (A2) by smoothness conditions of the function to be integrated to get the expansion of Theorem 2.1. Further, we have the analogous result to Theorem 2.11 of Götze and Hipp about the tail behaviour without Cramér's condition (A2).

(2) Usually, tapering causes a lot of technical trouble (cf. Dahlhaus (1983)). The proofs of the results given here need no special effort concerning tapering.

(3) Whereas in the cases of the estimates for the autocovariances (at different lags) and the Whittle estimates it is not difficult to fulfill the assumptions (A1) - (A5), in the cases of the estimates for the spectral distribution function and the spectral density function the assumption (A3) is hardly to verify. It is an open question if the assumption that the Fourier coefficients have to decay exponentially can be weakened and so Edgeworth expansions are valid at least for modified versions of the estimates mentioned (e.g. for smoothed versions and special kernels). 


\section{Whittle estimates}

Consider a linear process $\left\{\mathrm{X}_{\mathrm{t}}\right\}_{\mathrm{t} \in \mathbf{Z}}$ whose spectral density $\mathrm{f}_{\theta}$ can be parametrized by $\theta$ lying within a compact set $\Theta \subset \mathbf{R}$ (e.g. ARMA-processes). Assume that Kolmogorov's formula holds, i.e.

$$
\int_{-\pi}^{\pi} \log f_{\theta}(\alpha) d \alpha=2 \pi \log \frac{\sigma^{2}}{2 \pi}
$$

where $\sigma^{2}$ represents the innovation variance. For sake of simplicity we assume $\sigma^{2}$ to be known. Let $\theta_{0} \in$ Int $\Theta$ be the true, unknown parameter. Minimization of the function

$$
\mathrm{L}_{\mathrm{T}}(\theta) \equiv \int_{0}^{\pi} \mathrm{f}_{\theta}^{-1}(\alpha) \mathrm{I}_{\mathrm{T}}(\alpha) \mathrm{d} \alpha
$$

yields the well-known Whittle estimate $\widehat{\theta}$ for $\theta_{0}$. (cf. Dzhaparidze and Yaglom (1983)).

We give the Edgeworth expansion of the distribution of $\hat{\theta}$ up to second order and prove its validity.

First we set down the assumptions needed additional to the general assumptions (A1) to (A5).

(A6) The set of parameter $\Theta \subset \mathbf{R}$ is compact. The parameters are identifiable, i.e. $\theta_{1} \neq \theta_{2}$ implies $\mathrm{f}_{\theta_{1}} \neq \mathrm{f}_{\theta_{2}}$ on a set with positive Lebesgue measure.

The spectral density $\mathrm{f}_{\theta}(\alpha)$ is four times continuously differentiable with respect to $\theta \in \Theta$ and is two times continuously differentiable with respect to $\alpha \in[0, \pi] . f_{\theta}(\alpha)$ and its derivatives are uniformly bounded,

i.e. $\exists 0<\underline{\mathrm{c}} \leq \overline{\mathrm{c}}<\infty \quad \forall \theta \in \Theta, \alpha \in[0, \pi] \underline{\mathrm{c}} \leq \mathrm{f}_{\theta}(\alpha) \leq \overline{\mathrm{c}},\left|\frac{\partial}{\partial \theta^{(\mathrm{i})}} \mathrm{f}_{\theta}^{-1}(\alpha)\right| \leq \overline{\mathrm{c}}$, $\mathrm{i}=1, \ldots, 4$ and $\left|\frac{\partial}{\partial \alpha^{(j)}} \mathrm{f}_{\theta}(\alpha)\right| \leq \bar{c}, j=1,2$. Let $\phi_{\theta}=\left(\phi_{\theta}^{(1)}, \phi_{\theta}^{(2)}, \phi_{\theta}^{(3)}\right)$ with $\phi_{\theta}^{(i)} \equiv \frac{\partial}{\partial \theta^{(i)}} f_{\theta}^{-1}, i=1,2,3$. There exists $d_{0}>0$ such that $\mathrm{L}^{(2)}(\theta) \equiv \int_{0}^{\pi} \phi_{\theta}^{(2)}(\alpha) \mathrm{f}_{\theta}(\alpha) \mathrm{d} \alpha \geq \mathrm{d}_{0} \quad$ for all $\theta \in \Theta$ 
We now state the theorem.

\section{Theorem 3.1.}

Assume that (A1) - (A6) hold. Let $\alpha$ be an arbitrary fixed number such that $0<\alpha<1 / 4$.

(i) There exists a statistic $\hat{\theta}$ which solves

$$
\int_{0}^{\pi} \frac{\partial}{\partial \theta} \mathrm{f}_{\theta}^{-1}(\alpha) \mathrm{I}_{\mathrm{T}}(\alpha) \mathrm{d} \alpha=0
$$

such that for some $\mathrm{d}_{1}>0$

$$
\mathrm{P}_{\theta_{0}}\left(\left|\hat{\theta}-\theta_{0}\right|<\mathrm{d}_{1} \mathrm{~T}^{\alpha-1 / 2}\right)=1-\mathrm{o}\left(\mathrm{T}^{-1 / 2}\right)
$$

uniformly for $\theta_{0} \in \Theta$.

(ii) For $\hat{\theta}$ satisfying (3.4)

$\sup _{x \in \mathbf{R}} \mid P_{\theta_{0}}\left(\left(T K\left(\theta_{0}\right)\right){ }^{1 / 2}\left(\widehat{\theta}-\theta_{0}\right) \leq x\right)-\int_{-\infty}^{\mathrm{x}}\left(1+\mathrm{T}^{-1 / 2} \mathrm{p}_{3}(\mathrm{y}) \mathrm{d} \Phi(\mathrm{y}) \mid=\mathrm{o}\left(\mathrm{T}^{-1 / 2}\right)\right.$

uniformly for $\theta_{0} \in \Theta$, where $\mathrm{p}_{3}(\mathrm{x})$ denotes a polynomial in $x$ whose coefficients are continuous functions of the approximate cumulants of $\mathrm{U}_{\mathrm{T}}\left(\theta_{0}\right)$ (defined in (4.30)) of order three or less.

\section{Remark 3.2.}

(1) This result generalizes Theorem 3.2.1 by Taniguchi (1991) from Gaussian to linear processes.

(2) The Edgeworth expansion is valid up to higher order than given above

(cf. Taniguchi (1991)).

(3) The generalization to the multivariate case is not difficult, but requires cumbersome notations. 


\section{Proofs}

\section{Proof of Theorem 2.3.}

Conditions $\mathrm{C}(1)-\mathrm{C}(7)$ have to be verified. With $\mathcal{D}_{\mathrm{j}}=\sigma\left(\varepsilon_{\mathrm{j}}\right) \mathrm{C}(1), \mathrm{C}(4)$ and $\mathrm{C}(6)$ hold trivially. $\mathrm{C}(7)$ is assumption (A5). $\mathrm{C}(2)$ follows from

$$
\begin{aligned}
& \left(\mathbf{E}\left\|\mathrm{U}_{\mathrm{T}, \mathrm{t}}\right\|^{\mathrm{s}+1}\right)^{1 /(\mathrm{s}+1)} \\
& \quad \leq \sum_{\mathrm{r}=0}^{\mathrm{T}}\|\widehat{\psi}(\mathrm{r})\|\left|\mathrm{h}_{\mathrm{t}} \mathrm{h}_{\mathrm{t}+\mathrm{r}}\right| \sum_{\mathrm{u}, \mathrm{v} \in \mathbf{Z}}\left|\mathrm{a}_{\mathrm{t}-\mathrm{u}}\right|\left|\mathrm{a}_{\mathrm{t}+\mathrm{r}-\mathrm{v}}\right|\left(\mathbf{E}\left|\varepsilon_{\mathrm{u}} \varepsilon_{\mathrm{v}}\right|^{\mathrm{s}+1}\right)^{1 /(\mathrm{s}+1)} \\
& \quad \leq 2 \sum_{\mathrm{r} \in \mathbf{Z}}\|\widehat{\phi}(\mathrm{r})\|\left(\sum_{\mathrm{u} \in \mathbf{Z}}\left|\mathrm{a}_{\mathrm{u}}\right|\right)^{2}\left(\mathbf{E}\left|\varepsilon_{1}\right|^{2(\mathrm{~s}+1)}\right)^{1 /(\mathrm{s}+1)}<\infty
\end{aligned}
$$

by the assumptions (A1) and (A3).

To prove $\mathrm{C}(3)$ define $\mathrm{Y}_{\mathrm{T}, \mathrm{t}, \mathrm{m}} \in \mathcal{D}_{\mathrm{t}-\mathrm{m}}^{\mathrm{t}+\mathrm{m}}$ by

It suffices to show

$$
\mathrm{Y}_{\mathrm{T}, \mathrm{t}, \mathrm{m}} \equiv \sum_{\mathrm{r}=0}^{\mathrm{m}} \widehat{\psi}(\mathrm{r}) \mathrm{h}_{\mathrm{t}} \mathrm{h}_{\mathrm{t}+\mathrm{r}} \sum_{\substack{|\mathrm{t}-\mathrm{u}| \leq \mathrm{m} \\|\mathrm{t}-\mathrm{v}| \leq \mathrm{m}}} \mathrm{a}_{\mathrm{t}-\mathrm{u}} \mathrm{a}_{\mathrm{t}+\mathrm{r}-\mathrm{v}} \varepsilon_{\mathrm{u}} \varepsilon_{\mathrm{v}} .
$$

$$
\left(\mathbf{E}\left\|U_{T, t}-Y_{T, t, m}\right\|^{2}\right)^{1 / 2} \leq \rho^{m}
$$

First notice that the sum in the definition (2.1) of $\mathrm{U}_{\mathrm{T}, \mathrm{t}}$ can be restricted to the indices $\{0, \ldots,[\mathrm{m} / 2]\}$, since

$$
\begin{aligned}
\left(\mathbf{E}\left\|\sum_{\mathrm{r}>[\mathrm{m} / 2]} \hat{\psi}(\mathrm{r}) \mathrm{h}_{\mathrm{t}} \mathrm{h}_{\mathrm{t}+\mathrm{r}} X_{\mathrm{t}} \mathrm{X}_{\mathrm{t}+\mathrm{r}}\right\|^{2}\right)^{1 / 2} \\
\quad \leq 2 \sum_{\mathrm{r}>[\mathrm{m} / 2]}\|\phi(\mathrm{r})\|\left(\sum_{\mathrm{u} \in \mathbf{Z}}\left|\mathrm{a}_{\mathrm{u}}\right|^{2}\left(\mathbf{E}\left|\varepsilon_{1}\right|^{4}\right)^{1 / 4}\right. \\
\leq \frac{1}{2} \rho^{\mathrm{m}} \text { with } 0<\rho<1
\end{aligned}
$$

by assumption (A1) and the exponential decay of the coefficients $\|\phi(\mathrm{r})\|$ (see (A6)). 
Next, we compute the difference between the restricted sum, $\mathrm{U}_{\mathrm{T}, \mathrm{t}, \mathrm{m}}$ say, and $\mathrm{Y}_{\mathrm{T}, \mathrm{t}, \mathrm{m}}$.

$\left(\mathbf{E}\left\|\mathrm{U}_{\mathrm{T}, \mathrm{t}, \mathrm{m}}-\mathrm{Y}_{\mathrm{T}, \mathrm{t}, \mathrm{m}}\right\|^{2}\right)^{1 / 2}$

$$
\begin{aligned}
& \leq 2 \sum_{\mathrm{r}=0}^{[\mathrm{m} / 2]}\|\widehat{\phi}(\mathrm{r})\|\left(\mathbf{E}\left|\varepsilon_{1}\right|^{4}\right)^{1 / 4}\left(\sum_{|\mathrm{t}-\mathrm{u}| \leq \mathrm{m}}\left|\mathrm{a}_{\mathrm{t}-\mathrm{u}}\right| \sum_{|\mathrm{t}-\mathrm{v}|>\mathrm{m}}\left|\mathrm{a}_{\mathrm{t}+\mathrm{r}-\mathrm{v}}\right|+\sum_{|\mathrm{t}-\mathrm{u}|>\mathrm{m}}\left|\mathrm{a}_{\mathrm{t}-\mathrm{u}}\right| \sum_{\mathrm{v} \in \mathbf{Z}}\left|\mathrm{a}_{\mathrm{r}+\mathrm{t}-\mathrm{v} \mid}\right|\right) \\
& \leq 2 \sum_{\mathrm{r}=0}^{[\mathrm{m} / 2]}\|\widehat{\phi}(\mathrm{r})\|\left(\mathbf{E}\left|\varepsilon_{1}\right|^{4}\right)^{1 / 4}\left(\sum_{\mathrm{u} \in \mathbf{Z}}\left|\mathrm{a}_{\mathrm{u}}\right| \sum_{|\mathrm{v}|>[\mathrm{m} / 2]}\left|\mathrm{a}_{\mathrm{v}}\right|+\sum_{|\mathrm{u}|>\mathrm{m}}\left|\mathrm{a}_{\mathrm{u}}\right| \sum_{\mathrm{v} \in \mathbf{Z}}\left|\mathrm{a}_{\mathrm{v}}\right|\right) \leq \frac{1}{2} \rho^{\mathrm{m}}
\end{aligned}
$$

by the assumption (A1) and the exponential decay of the coefficients $\left|a_{\mathrm{u}}\right|((\mathrm{A} 3))$ ). (4.3) and (4.4) implies (4.2). It remains to check the Cramér type condition $\mathrm{C}(5)$.

$$
\begin{aligned}
\sum_{|t-j| \leq m} U_{T, j} & =\sum_{u, v \in Z} \varepsilon_{u} \varepsilon_{v} \sum_{|t-j| \leq m} \sum_{r=0}^{T} \widehat{\psi}(r) h_{j} h_{j+r} a_{j-u} a_{j+r-v} \\
& =\varepsilon_{t} A_{T, t, m}+\varepsilon_{t}^{2} B_{T, t, m}+\zeta
\end{aligned}
$$

where

$$
\begin{gathered}
A_{T, t, m} \equiv \sum_{|j| \leq m} \sum_{r=0}^{T} \widehat{\psi}(r) h_{t+j} h_{t+j+r}\left(a_{j} \sum_{v \neq t} a_{t+j+r-v} \varepsilon_{v}+a_{j+r} \sum_{u \neq t} a_{t+j-u} \varepsilon_{u}\right), \\
B_{T, t, m} \equiv \sum_{|j| \leq m} \sum_{r=0}^{T} \widehat{\psi}(r) h_{t+j} h_{t+j+r} a_{j} a_{j+r}
\end{gathered}
$$

and $\zeta$ denotes a random vector stochastically independent of $\varepsilon_{\mathrm{t}}$. Note that $\mathrm{A}_{\mathrm{T}, \mathrm{t}, \mathrm{m}}$ and $\varepsilon_{\mathrm{t}}$ are also independent for all t. Let $\left\{\varepsilon_{\mathrm{j}}^{*}\right\}$ be i.i.d. rvs, $\left\{\varepsilon_{\mathrm{j}}^{*}\right\}$ and $\left\{\varepsilon_{\mathrm{j}}\right\}$ independent and $\varepsilon_{\mathrm{j}}^{*} \stackrel{\mathcal{D}}{=} \varepsilon_{\mathrm{j}}$. Define $\mathrm{A}_{\mathrm{T}, \mathrm{t}, \mathrm{m}}^{*}$ as $\mathrm{A}_{\mathrm{T}, \mathrm{t}, \mathrm{m}}$ with $\varepsilon_{\mathrm{j}}^{\prime} \mathrm{s}$ replaced by $\varepsilon_{\mathrm{j}}^{*_{1}} \mathrm{~s}$. 
Thus, with $\theta \in \mathbf{R}^{\mathrm{d}}$

$$
\begin{aligned}
& \mathbf{E}\left|\mathbf{E} \exp \left(\mathrm{i} \theta^{\prime} \sum_{|\mathrm{t}-\mathrm{j}| \leq \mathrm{m}} \mathrm{Z}_{\mathrm{T}, \mathrm{j}} \mid \mathcal{D}_{\mathrm{j}}: \mathrm{j} \neq \mathrm{t}\right)\right| \\
& =\mathbf{E}\left|\mathbf{E} \exp \left(\mathrm{i} \varepsilon_{\mathrm{t}} \theta^{\prime} \mathrm{A}_{\mathrm{T}, \mathrm{t}, \mathrm{m}}+\mathrm{i} \varepsilon_{\mathrm{t}}^{2} \theta^{\prime} \mathrm{B}_{\mathrm{T}, \mathrm{t}, \mathrm{m}} \mid \mathcal{D}_{\mathrm{j}}: \mathrm{j} \neq \mathrm{t}\right)\right| \\
& =\mathbf{E}\left|\mathbf{E} \exp \left(\mathrm{i} \varepsilon_{\mathrm{t}} \theta^{\prime} \mathrm{A}_{\mathrm{T}, \mathrm{t}, \mathrm{m}}^{*}+\mathrm{i} \varepsilon_{\mathrm{t}}^{2} \theta^{\prime} \mathrm{B}_{\mathrm{T}, \mathrm{t}, \mathrm{m}}\right)\right| \\
& \leq(1-\delta) \mathrm{P}\left(\left\|\theta^{\prime} \mathrm{A}_{\mathrm{T}, \mathrm{t}, \mathrm{m}}^{*}, \theta^{\prime} \mathrm{B}_{\mathrm{T}, \mathrm{t}, \mathrm{m}}\right\| \geq \mathrm{d}\right) \\
& \quad+\mathrm{P}\left(\left\|\theta^{\prime} \mathrm{A}_{\mathrm{T}, \mathrm{t}, \mathrm{m}}^{*}, \theta^{\prime} \mathrm{B}_{\mathrm{T}, \mathrm{t}, \mathrm{m}}\right\|<\mathrm{d}\right)
\end{aligned}
$$

by Cramér's condition on $\left(\varepsilon_{\mathrm{t}}, \varepsilon_{\mathrm{t}}^{2}\right)$ (see (A2)).

Hence $C(5)$ will follow if constants $d, d_{1}, \eta>0$ exist such that for $\|\theta\| \geq d_{1}$

$$
\mathrm{P}\left(\left\|\theta^{\prime} \mathrm{A}_{\mathrm{T}, \mathrm{t}, \mathrm{m}}^{*}, \theta^{\prime} \mathrm{B}_{\mathrm{T}, \mathrm{t}, \mathrm{m}}\right\| \geq \mathrm{d}\right)>\eta
$$

holds for a sufficiently large number of t's. This is verified, if there exists $\varepsilon, \eta>0$ such that for all $\|\theta\|=1$,

$$
\mathrm{P}\left(\left\|\theta^{\prime} \mathrm{A}_{\mathrm{T}, \mathrm{t}, \mathrm{m}}, \theta^{\prime} \mathrm{B}_{\mathrm{T}, \mathrm{t}, \mathrm{m}}\right\| \geq \varepsilon\right)>\eta
$$

holds. But this is lemma 4.2. Thus $\mathrm{C}(5)$ is fulfilled and the theorem follows.

First, we set down another lemma which will be needed.

\section{Lemma 4.1.}

Assume the conditions of Theorem 2.3. Then

(i)

$$
\begin{aligned}
& \mathrm{c}_{\mathrm{T}}^{-1} \sum_{\mathrm{t}=1}^{\mathrm{T}} \mathrm{D}\left(\mathrm{A}_{\mathrm{T}, \mathrm{t}, \mathrm{m}}\right) \rightarrow \mathrm{D}(\mathrm{A}) \quad \text { for } \mathrm{m}<\mathrm{T} \text { and } \mathrm{m} \rightarrow \infty, \\
& \text { where } \mathrm{A} \equiv 2 \frac{\mathrm{H}_{4}^{1 / 2}}{\mathrm{H}_{2}} \sum_{\mathrm{u} \neq 0} \varepsilon_{\mathrm{u}} \int \phi(\alpha) \mathrm{f}(\alpha) \cos (\alpha \mathrm{u}) \mathrm{d} \alpha,
\end{aligned}
$$


(ii)

$$
\begin{aligned}
& \mathrm{D}(\mathrm{A})=2\left(\sum+\mathrm{C}_{0} \mathrm{~N}\right) \\
& \text { where } \mathrm{C}_{0} \equiv 1-\mathbf{E} \varepsilon_{1}^{4}<0, \\
& \mathrm{~N} \equiv \frac{\mathrm{H}_{4}}{\mathrm{H}_{2}^{2}} \int \phi \mathrm{f} \int \phi^{\prime} \mathrm{f} \\
& \text { and } \quad \equiv \frac{\mathrm{H}_{4}}{\mathrm{H}_{2}^{2}} \cdot 2 \pi \int \phi \phi^{\prime} \mathrm{f}^{2}+\left(\mathbf{E} \varepsilon_{1}^{4}-3\right) \mathrm{N} . \\
& \frac{1}{2 \pi \mathrm{H}_{2, \mathrm{~T}}} \sum_{\mathrm{t}=1}^{\mathrm{T}} \mathrm{B}_{\mathrm{T}, \mathrm{t}, \mathrm{m}} \mathrm{B}_{\mathrm{T}, \mathrm{t}, \mathrm{m}}^{\prime}
\end{aligned}
$$

(iii)

\section{Proof.}

Ad (i). First, we give a simplification for $\mathrm{A}_{T, t, \mathrm{~m}}$.

$$
\begin{aligned}
a_{j} \sum_{v \neq t} a_{t+j+r-v} & \varepsilon_{v}+a_{j+r} \sum_{u \neq t} a_{t+j-u} \varepsilon_{u} \\
= & a_{j} X_{t+j+r}+a_{j+r} X_{t+j}-2 a_{j} a_{j+r} \varepsilon_{t} \\
= & a_{j} \sum_{u \in Z} a_{j+r-u} \varepsilon_{t+u}+a_{j+r} \sum_{u \in Z} a_{j-u} \varepsilon_{t+u}-2 a_{j} a_{j+r} \varepsilon_{t} \\
= & \sum_{u \neq 0} \varepsilon_{t+u}\left(a_{j} a_{j+r-u}+a_{j+r} a_{j-u}\right) .
\end{aligned}
$$

Thus

$$
\begin{aligned}
A_{T, t, m} & =\sum_{|j| \leq m} \sum_{r=0}^{T} \widehat{\psi}(r) h_{t+j} h_{t+j+r} \sum_{u \neq 0} \varepsilon_{t+u}\left(a_{j} a_{j+r-u}+a_{j+r} a_{j-u}\right) \\
& =\sum_{u \neq 0} \varepsilon_{t+u} \sum_{r=0}^{T} \widehat{\psi}(r)\left(a_{T, t, m}^{-}(r, u)+a_{T, t, m}^{+}(r, u)\right),
\end{aligned}
$$

where

$$
a_{T, t, m}^{-}(r, u) \equiv \sum_{|j| \leq m} h_{t+j} h_{t+j+r} a_{j} a_{j+r-u},
$$




$$
a_{T, t, m}^{+}(r, u) \equiv \sum_{|j| \leq m} h_{t+j} h_{t+j+r} a_{j+r} a_{j-u}
$$

Next, we calculate products of the terms in (4.11) and (4.12). We have

$\sum_{\mathrm{t}=1}^{\mathrm{T}} \mathrm{a}_{\mathrm{T}, \mathrm{t}, \mathrm{m}}^{\mathrm{o}}(\mathrm{r}, \mathrm{u}) \mathrm{a}_{\mathrm{T}, \mathrm{t}, \mathrm{m}}^{\Delta}(\mathrm{s}, \mathrm{u})=\left(\mathrm{H}_{4, \mathrm{~T}}+\mathrm{O}(|\mathrm{r}|+|\mathrm{s}|)\right)\left(\mathrm{c}(\mathrm{r} \circ \mathrm{u}) \mathrm{c}(\mathrm{s} \Delta \mathrm{u})+\mathrm{O}\left(\rho^{\mathrm{m}}\right)\right)$,

where $c(u)=\sum_{j \in \mathbf{Z}} a_{j} a_{j+u} \quad$ and $o, \Delta \in\{+,-\}$.

We consider the case $\mathrm{o}=-, \Delta=+$.

$$
\begin{aligned}
\sum_{t=1}^{T} a_{T, t, m}^{-}(r, u) a_{T, t, m}^{+}(s, u) & =\sum_{|j|,|k| \leq m} a_{j} a_{j+r-u} a_{k+s} a_{k-u} \sum_{t=1}^{T} h_{t+j} h_{t+j+r} h_{t+k} h_{t+k+s} \\
& =\sum_{|j|,|k| \leq m} a_{j} a_{j+r-u} a_{k+s} a_{k-u}\left(H_{4, T}+O(|j|+|k|+|r|+|s|)\right)
\end{aligned}
$$

by lemma P 4.1 in Brillinger (1981).

Now (4.13) follows from assumption (A3) on the coefficients $\left\{a_{j}\right\}$.

From (4.10) and (4.13) we get

$$
\begin{aligned}
& \mathrm{c}_{\mathrm{T}}^{-1} \sum_{\mathrm{t}=1}^{\mathrm{T}} \mathrm{D}\left(\mathrm{A}_{\mathrm{T}, \mathrm{t}, \mathrm{m}}\right) \\
& =\mathrm{c}_{\mathrm{T}}^{-1} \sum_{\mathrm{t}=1}^{\mathrm{T}} \sum_{\mathrm{u}, \mathrm{v} \neq 0} \mathbf{E} \varepsilon_{\mathrm{t}+\mathrm{u}} \varepsilon_{\mathrm{t}+\mathrm{v}} \sum_{\mathrm{r}, \mathrm{s}=0}^{\mathrm{T}} \widehat{\psi}(\mathrm{r}) \widehat{\psi}^{\prime}(\mathrm{s})\left(\mathrm{a}_{\mathrm{T}, \mathrm{t}, \mathrm{m}}^{-}(\mathrm{r}, \mathrm{u})+\mathrm{a}_{\mathrm{T}, \mathrm{t}, \mathrm{m}}^{+}(\mathrm{r}, \mathrm{u})\right) \\
& \quad\left(\mathrm{a}_{\mathrm{T}, \mathrm{t}, \mathrm{m}}^{-}(\mathrm{s}, \mathrm{v})+\mathrm{a}_{\mathrm{T}, \mathrm{t}, \mathrm{m}}^{+}(\mathrm{s}, \mathrm{v})\right) \\
& =\mathrm{c}_{\mathrm{T}}^{-1} \sum_{\mathrm{u} \neq 0} \sum_{\mathrm{r}, \mathrm{s}=0}^{\mathrm{T}} \widehat{\psi}(\mathrm{r}) \widehat{\psi}^{\prime}(\mathrm{s}) \sum_{\mathrm{t}=1}^{\mathrm{T}}\left(\mathrm{a}_{\mathrm{T}, \mathrm{t}, \mathrm{m}}^{-}(\mathrm{s}, \mathrm{u})+\mathrm{a}_{\mathrm{T}, \mathrm{t}, \mathrm{m}}^{+}(\mathrm{r}, \mathrm{u})\right)\left(\mathrm{a}_{\mathrm{T}, \mathrm{t}, \mathrm{m}}^{-}(\mathrm{s}, \mathrm{u})+\mathrm{a}_{\mathrm{T}, \mathrm{t}, \mathrm{m}}^{+}(\mathrm{s}, \mathrm{u})\right)
\end{aligned}
$$




$$
\begin{aligned}
& =\mathrm{H}_{4, \mathrm{~T}} \mathrm{c}_{\mathrm{T}}^{-1} \sum_{\mathrm{u} \neq 0} \sum_{|\mathrm{r}| \leq \mathrm{T}} \hat{\phi}(\mathrm{r})(\mathrm{c}(\mathrm{r}+\mathrm{u})+\mathrm{c}(\mathrm{r}-\mathrm{u})) \sum_{|\mathrm{s}| \leq \mathrm{T}} \hat{\phi}^{\prime}(\mathrm{s})(\mathrm{c}(\mathrm{s}+\mathrm{u})+\mathrm{c}(\mathrm{s}-\mathrm{u}))+\mathrm{O}\left(\rho^{\mathrm{m}}\right) \\
& \rightarrow \frac{\mathrm{H}_{4}}{\mathrm{H}_{2}^{2}} \sum_{\mathrm{u} \neq 0} \int \phi(\alpha) \mathrm{f}(\alpha)\left(\mathrm{e}^{\mathrm{i} \alpha \mathrm{u}}+\mathrm{e}^{-\mathrm{i} \alpha \mathrm{u}}\right) \mathrm{d} \alpha \int \phi^{\prime}(\alpha) \mathrm{f}(\alpha)\left(\mathrm{e}^{\mathrm{i} \alpha \mathrm{u}}+\mathrm{e}^{-\mathrm{i} \alpha \mathrm{u}}\right) \mathrm{d} \alpha
\end{aligned}
$$

for $\mathrm{m}<\mathrm{T}$ and $\mathrm{m} \rightarrow \infty$ by Parseval's identity.

Ad (ii).

$$
\begin{aligned}
D(A)= & 4 \frac{H_{4}}{H_{2}^{2}} \sum_{u, v \neq 0} \int \phi(\alpha) f(\alpha) \cos (\alpha u) d \alpha \int \phi^{\prime}(\alpha) f(\alpha) \cos (\alpha u) d \alpha \mathbf{E} \varepsilon_{\mathrm{u}} \varepsilon_{\mathrm{v}} \\
= & 4 \frac{\mathrm{H}_{4}}{\mathrm{H}_{2}^{2}}\left(\sum_{\mathrm{u} \in \mathbf{Z}} \int \phi(\alpha) \mathrm{f}(\alpha) \cos (\alpha \mathrm{u}) \mathrm{d} \alpha \int \phi^{\prime}(\alpha) \mathrm{f}(\alpha) \cos (\alpha \mathrm{u}) \mathrm{d} \alpha\right. \\
& \left.-\int \phi(\alpha) \mathrm{f}(\alpha) \mathrm{d} \alpha \int \phi^{\prime}(\alpha) \mathrm{f}(\alpha) \mathrm{d} \alpha\right) \\
= & 2 \cdot \frac{\mathrm{H}_{4}}{\mathrm{H}_{2}^{2}}\left(2 \pi \int \phi(\alpha) \phi^{\prime}(\alpha) \mathrm{f}^{2}(\alpha) \mathrm{d} \alpha-2 \cdot \int \phi(\alpha) \mathrm{f}(\alpha) \mathrm{d} \alpha \int \phi^{\prime}(\alpha) \mathrm{f}(\alpha) \mathrm{d} \alpha\right)
\end{aligned}
$$

by Parseval's identity.

Ad (iii).

The proof is analogous to (i), but much simpler, and therefore omitted.

\section{Lemma 4.2.}

Assume the conditions of Theorem 2.3. Then

$$
\begin{aligned}
& \exists \varepsilon, \eta, \rho>0 \quad \forall\|\theta\|=1 \quad \forall \rho^{-1}<\mathrm{m}<\mathrm{T} \\
& \#\left\{\mathrm{t} \in\{1, \ldots, \mathrm{T}\}: \quad \mathrm{P}\left(\left\|\theta^{\prime} \mathrm{A}_{\mathrm{T}, \mathrm{t}, \mathrm{m}}, \theta^{\prime} \mathrm{B}_{\mathrm{T}, \mathrm{t}, \mathrm{m}}\right\| \geq \varepsilon\right)>\eta\right\} \geq \rho \mathrm{T} .
\end{aligned}
$$

\section{Proof.}

From the compactness of the unit ball, it suffices to show that there is such a choice of $\varepsilon$ and $\eta$ for every fixed $\theta$. Choose such a $\theta$ and write $\theta=\theta_{1}+\theta_{2}$, where $\theta_{1}$ is orthogonal to $\theta_{2}$ and $\theta_{1}$ $=\mathrm{c} \int \phi(\alpha) \mathrm{f}(\alpha) \mathrm{d} \alpha$ for some c. Fix $\alpha>0$ (to be chosen). 


\section{Case 1.}

$\left\|\theta_{1}\right\| \geq \alpha$. Then

$$
\left\|\theta^{\prime} \mathrm{A}_{\mathrm{T}, \mathrm{t}, \mathrm{m}}, \theta^{\prime} \mathrm{B}_{\mathrm{T}, \mathrm{t}, \mathrm{m}}\right\| \geq\left|\theta^{\prime} \mathrm{B}_{\mathrm{T}, \mathrm{t}, \mathrm{m}}\right|
$$

By Cauchy-Schwartz inequality we find a positive constant a only depending on the coefficients $\left\{\mathrm{a}_{\mathrm{j}}\right\}$ and $\{\|\hat{\psi}(\mathrm{r})\|\}$ with

$$
\left|\theta^{\prime} \mathrm{B}_{\mathrm{T}, \mathrm{t}, \mathrm{m}}\right|^{2} \leq \mathrm{a}
$$

Lemma 4.1 (iii) delivers

$$
\begin{aligned}
\frac{1}{2 \pi \mathrm{H}_{2, \mathrm{~T}}} \sum_{\mathrm{t}=1}^{\mathrm{T}}\left|\theta^{\prime} \mathrm{B}_{\mathrm{T}, \mathrm{t}, \mathrm{m}}\right|^{2} & =\theta^{\prime} \frac{1}{2 \pi \mathrm{H}_{2, \mathrm{~T}}} \sum_{\mathrm{t}=1}^{\mathrm{T}} \mathrm{B}_{\mathrm{T}, \mathrm{t}, \mathrm{m}} \mathrm{B}_{\mathrm{T}, \mathrm{t}, \mathrm{m}}^{\prime} \theta \\
& \rightarrow \theta^{\mathrm{m}} \rightarrow \infty \\
& =\frac{\mathrm{H}_{4}}{\mathrm{H}_{2}^{2}}\left|\theta_{1}^{\prime} \int \phi \mathrm{f}\right|^{2} \\
& \geq \alpha^{2} \frac{\mathrm{H}_{4}}{\mathrm{H}_{2}^{2}}\left\|\int \phi \mathrm{f}\right\|^{2} \equiv \mathrm{b}>0 .
\end{aligned}
$$

Assume w.1.o.g. $a \geq \max (b, 1)$. Let $c=\frac{b}{a} \leq 1$ and $\rho=\frac{c-\varepsilon}{1-\varepsilon}$. If less than $\rho \cdot T$ terms had the property $\left|\theta^{\prime} \mathrm{B}_{\mathrm{T}, \mathrm{t}, \mathrm{m}}\right|^{2} \geq \varepsilon$, we could bound the left-hand side of (4.16) by

$$
\begin{aligned}
\frac{1}{2 \pi \mathrm{H}_{2, \mathrm{~T}}} \sum_{\mathrm{t}=1}^{\mathrm{T}}\left|\theta^{\prime} \mathrm{B}_{\mathrm{T}, \mathrm{t}, \mathrm{m}}\right|^{2} & <(1-\rho) \varepsilon+\rho \mathrm{a} \\
& \leq \mathrm{a}((1-\rho) \varepsilon+\rho) \\
& =\mathrm{a}(\varepsilon+(1-\varepsilon) \rho) \\
& =\mathrm{b},
\end{aligned}
$$

which is a contradiction to (4.16). 


\section{Case 2.}

$\left\|\theta_{1}\right\| \geq 1-\alpha$. In this case

$$
\left\|\theta^{\prime} \mathrm{A}_{\mathrm{T}, \mathrm{t}, \mathrm{m}}, \theta^{\prime} \mathrm{B}_{\mathrm{T}, \mathrm{t}, \mathrm{m}}\right\| \geq\left|\theta^{\prime} \mathrm{A}_{\mathrm{T}, \mathrm{t}, \mathrm{m}}\right| .
$$

Cauchy-Schwartz inequality provides for

$$
\mathbf{E}\left|\theta^{\prime} \mathrm{A}_{\mathrm{T}, \mathrm{t}, \mathrm{m}}\right|^{2} \leq \mathrm{a}
$$

with a being a positive constant only depending on the coefficients $\left\{\mathrm{a}_{\mathrm{j}}\right\},\{\|\widehat{\psi}(\mathrm{r})\|\}$ and $\mathbf{E} \varepsilon_{1}^{2}$.

By lemma 4.1 (i) and (ii) we have

$$
\begin{aligned}
c_{\mathrm{T}}^{-1} \sum_{\mathrm{t}=1}^{\mathrm{T}} \mathbf{E}\left|\theta^{\prime} \mathrm{A}_{\mathrm{T}, \mathrm{t}, \mathrm{m}}\right|^{2} & =\theta^{\prime} \mathrm{c}_{\mathrm{T}}^{-1} \sum_{\mathrm{t}=1}^{\mathrm{T}} \mathrm{D}\left(\mathrm{A}_{\mathrm{T}, \mathrm{t}, \mathrm{m}}\right) \theta \\
& \stackrel{\mathrm{m} \rightarrow \infty}{\rightarrow} \theta^{\prime} \mathrm{D}(\mathrm{A}) \theta \\
& =2 \theta^{\prime}\left(\sum+\mathrm{C}_{0} \mathrm{~N}\right) \theta .
\end{aligned}
$$

Let $\lambda_{1}$ be the smallest eigenvalue of $\sum\left(\lambda_{1}>0\right.$ !). Then we can continue

$$
\geq 2\left(\lambda_{1}-\left|\mathrm{C}_{0}\right| \theta^{\prime} \mathrm{N} \quad \theta\right) \geq \lambda_{1}>0,
$$

if $\left|\mathrm{C}_{0}\right| \quad \theta^{\prime} \mathrm{N} \quad \theta \leq \lambda_{1}$.

But, by Cauchy Schwartz inequality

$$
\begin{aligned}
\left|\mathrm{C}_{0}\right| \theta^{\prime} \mathrm{N} \theta & =\frac{\mathrm{H}_{4}}{\mathrm{H}_{2}^{2}}\left|\theta_{1}^{\prime} \int \phi \mathrm{f}\right|^{2} \\
& \leq \alpha^{2}\left|\mathrm{C}_{0}\right| \frac{\mathrm{H}_{4}}{\mathrm{H}_{2}^{2}}\left\|\int \phi \mathrm{f}\right\|^{2}
\end{aligned}
$$


and thus (4.18) holds with an $\alpha$ chosen sufficiently small. Now the assertion follows from (4.17) and (4.18) as in case 1. This proves the lemma.

Before proving Theorem 3.1, we state some preparations and several lemmas. We set down

$$
\begin{aligned}
\mathrm{L}^{(\mathrm{i})}(\theta) & \equiv \int_{0}^{\pi} \phi_{\theta}^{(\mathrm{i})}(\alpha) \mathrm{f}_{\theta}(\alpha) \mathrm{d} \alpha \\
\mathrm{L}_{\mathrm{T}}^{(\mathrm{i})}(\theta) & \equiv \int_{0}^{\pi} \phi_{\theta}^{(\mathrm{i})}(\alpha) \mathrm{I}_{\mathrm{T}}(\alpha) \mathrm{d} \alpha \\
\mathrm{Z}_{\mathrm{i}}(\theta) & \equiv \sqrt{\mathrm{T}}\left(\mathrm{L}_{\mathrm{T}}^{(\mathrm{i})}(\theta)-\mathbf{E} \mathrm{L}_{\mathrm{T}}^{(\mathrm{i})}(\theta)\right)
\end{aligned}
$$

for $\mathrm{i}=1,2,3$.

\section{Lemma 4.3.}

Under (A1), (A3), (A4) and (A6)

$$
\mathbf{E}_{\theta} \mathrm{L}_{\mathrm{T}}^{(\mathrm{i})}(\theta)=\mathrm{L}^{(\mathrm{i})}(\theta)+\mathrm{o}\left(\mathrm{T}^{-1}\right), \mathrm{i}=1,2,3
$$

$$
\mathbf{E}_{\theta}\left(\mathrm{Z}_{1}(\theta)\right)^{2}=\frac{\mathrm{H}_{4}}{\mathrm{H}_{2}^{2}} 2 \pi \int_{0}^{\pi}\left(\phi_{\theta}^{(1)}(\alpha) \mathrm{f}_{\theta}(\alpha)\right)^{2} \mathrm{~d} \alpha+\mathrm{o}(1)
$$

$$
\mathbf{E}_{\theta}\left(Z_{1}(\theta) Z_{2}(\theta)\right)=\frac{H_{4}}{H_{2}^{2}} 2 \pi \int_{0}^{\pi} \phi_{\theta}^{(1)}(\alpha) \phi_{\theta}^{(2)}(\alpha) f_{\theta}^{2}(\alpha) d \alpha+o(1)
$$

$$
\sqrt{T} \mathbf{E}_{\theta}\left(\mathrm{Z}_{1}(\theta)\right)^{3}=\frac{\mathrm{H}_{6}}{\mathrm{H}_{2}^{3}} 8 \pi^{2} \int_{0}^{\pi}\left(\phi_{\theta}^{(1)}(\alpha) \mathrm{f}_{\theta}(\alpha)\right)^{3} \mathrm{~d} \alpha+\mathrm{o}(1)
$$

uniformly for $\theta \in \Theta$. 
For the proof we refer to Dahlhaus (1983).

\section{Lemma 4.4.}

Under (A1) - (A6), for every $\alpha>0$ and some $\mathrm{d}_{2}>0$, we have

$$
\mathrm{P}_{\theta}\left(\left|\mathrm{Z}_{\mathrm{i}}(\theta)\right|>\mathrm{d}_{2} \mathrm{~T}^{\alpha}\right)=\mathrm{o}\left(\mathrm{T}^{-1 / 2}\right), \quad \mathrm{i}=1,2,3
$$

uniformly for $\theta \in \Theta$.

The lemma is a direct consequence of Theorem 2.3.

The following result is due to Chibisov (1972).

\section{Lemma 4.5.}

Let $\mathrm{Y}_{\mathrm{T}}$ be a random variable which has the stochastic expansion

$$
\mathrm{Y}_{\mathrm{T}}=\mathrm{Y}_{\mathrm{T}}^{(3)}+\mathrm{T}^{-1} \xi_{\mathrm{T}}
$$

where the distribution of $\mathrm{Y}_{\mathrm{T}}^{(3)}$ has the Edgeworth expansion:

$$
\mathbf{P}\left(\mathrm{Y}_{\mathrm{T}}^{(3)} \leq \mathrm{x}\right)=\int_{0}^{\mathrm{x}}\left(1+\mathrm{T}^{-1 / 2} \mathrm{p}_{3}(\mathrm{y})\right) \mathrm{d} \Phi(\mathrm{y})+\mathrm{o}\left(\mathrm{T}^{-1 / 2}\right)
$$

Also $\xi_{\mathrm{T}}$ satisfies

$$
\left.\mathrm{P}\left(\mid \xi_{\mathrm{T}}\right)>\rho_{\mathrm{T}} \sqrt{\mathrm{T}}\right)=\mathrm{o}\left(\mathrm{T}^{-1 / 2}\right),
$$

where $\rho_{\mathrm{T}} \rightarrow 0, \rho_{\mathrm{T}} \sqrt{\mathrm{T}} \rightarrow \infty \quad$ as $\quad \mathrm{T} \rightarrow \infty$. Then

$$
\mathrm{P}\left(\mathrm{Y}_{\mathrm{T}} \leq \mathrm{x}\right)=\int_{-\infty}^{\mathrm{x}}\left(1+\mathrm{T}^{-1 / 2} \mathrm{p}_{3}(\mathrm{y})\right) \mathrm{d} \Phi(\mathrm{y})+\mathrm{o}\left(\mathrm{T}^{-1 / 2}\right)
$$


We return to the proof of (i) in Theorem 3.1.

\section{Proof of (i) in Theorem 3.1.}

We use the argument similar to that of Taniguchi (1991). Consider the equation

$$
0=\mathrm{L}_{\mathrm{T}}^{(1)}\left(\theta_{0}\right)+\mathrm{L}_{\mathrm{T}}^{(2)}\left(\theta_{0}\right)\left(\theta-\theta_{0}\right)+\frac{1}{2} \mathrm{~L}_{\mathrm{T}}^{(3)}\left(\theta_{0}\right)\left(\theta-\theta_{0}\right)^{2}+\mathrm{R}_{\mathrm{T}}(\theta)
$$

where

$$
\left|\mathrm{R}_{\mathrm{T}}(\theta)\right| \leq \frac{1}{6} \cdot \sup _{\left|\theta^{\prime}-\theta\right| \leq\left|\theta-\theta_{0}\right|}\left|\mathrm{L}_{\mathrm{T}}^{(4)}\left(\theta^{\prime}\right)\right|\left|\theta-\theta_{0}\right|^{3}
$$

For every $\alpha>0$ there exists a positive constant $d_{4}$ such that

$$
\mathbf{P}_{\theta_{0}}\left(\left|\mathrm{R}_{\mathrm{T}}(\theta)\right|>\left|\theta-\theta_{0}\right|^{3} \mathrm{~d}_{4} \mathrm{~T}^{\alpha}\right)=\mathrm{o}\left(\mathrm{T}^{-1 / 2}\right)
$$

For the proof of (4.27) notice

$$
\begin{aligned}
& \mathbf{P}_{\theta_{0}}\left(\sup _{\theta \in \Theta}\left|\mathrm{L}_{\mathrm{T}}^{(4)}(\theta)\right|>\mathrm{dT}^{\alpha}\right) \\
& \quad \leq \mathbf{P}_{\theta_{0}}\left(\sup _{\theta \in \Theta} \sup _{\lambda \in[0, \pi]}\left|\frac{\partial}{\partial \theta^{4}} \mathrm{f}_{\theta}^{-1}(\lambda)\right| \int_{0}^{\pi} \mathrm{I}_{\mathrm{T}}(\lambda) \mathrm{d} \lambda>\mathrm{dT}^{\alpha}\right) \\
& \quad \leq \mathbf{P}_{\theta_{0}}\left(\int_{0}^{\pi} \mathrm{I}_{\mathrm{T}}(\lambda) \mathrm{d} \lambda>\frac{\mathrm{d}}{\overline{\mathrm{c}}} \mathrm{T}^{\alpha}\right)
\end{aligned}
$$

by (A6). But the last term is of the order $\mathrm{o}\left(\mathrm{T}^{-1 / 2}\right)$ by Theorem 2.3. Therefore, on a set having $\mathbf{P}_{\theta_{0}}$-probability at least $1-\mathrm{o}\left(\mathrm{T}^{-1 / 2}\right)$, for some constants $\mathrm{d}_{5}>0$ and $\mathrm{d}_{4}>0$ we can rewrite (4.25) as

$$
\theta-\theta_{0}=\left(\mathrm{I}\left(\theta_{0}\right)+\eta_{\mathrm{T}}\right)^{-1}\left(\delta_{\mathrm{T}}+1 / 2 \mathrm{~L}_{\mathrm{T}}^{(3)}\left(\theta_{0}\right)\left(\theta-\theta_{0}\right)^{2}+\mathrm{d}_{5}\left|\theta-\theta_{0}\right|^{3} \xi_{\mathrm{T}}\right)
$$


where $\eta_{\mathrm{T}}$ and $\delta_{\mathrm{T}}$ are random variables whose absolute values are less than $\mathrm{d}_{6} \mathrm{~T}^{-1 / 2+\alpha}$ and $\xi_{\mathrm{T}}$ is a random variable whose absolute value is less then $\mathrm{d}_{4} \mathrm{~T}^{\alpha}$. There exist a sufficiently large $\mathrm{d}_{7}>0$ and an integer $\mathrm{T}_{0}$ such that if $\mathrm{T}>\mathrm{T}_{0}$ and $\left|\theta-\theta_{0}\right| \leq \mathrm{d}_{7} \mathrm{~T}^{-1 / 2+\alpha}(0<\alpha<1 / 4)$, the righthand side of (4.28) is less than $\mathrm{d}_{7} \mathrm{~T}^{-1 / 2+\alpha}$. Applying the Brower fixed point theorem to the right-hand side of (4.28) we have proved (i) of Theorem 3.1.

Now we set down

$$
\mathrm{V}_{\mathrm{T}} \equiv \sqrt{\mathrm{T}}\left(\widehat{\theta}-\theta_{0}\right)
$$

and

$$
\mathrm{U}_{\mathrm{T}}(\theta)=-\frac{\mathrm{Z}_{1}(\theta)}{\mathrm{L}^{(2)}(\theta)}+\frac{1}{\sqrt{\mathrm{T}}} \frac{\mathrm{Z}_{1}(\theta)}{\mathrm{L}^{(2)}(\theta)} \frac{\mathrm{Z}_{2}(\theta)}{\mathrm{L}^{(2)}(\theta)}-\frac{1}{2 \sqrt{\mathrm{T}}} \frac{\mathrm{L}^{(3)}(\theta)}{\mathrm{L}^{(2)}(\theta)}\left(\frac{\mathrm{Z}_{1}(\theta)}{\mathrm{L}^{(2)}(\theta)}\right)^{2}
$$

\section{Lemma 4.6.}

Under (A1) - (A6) we have the following stochastic expansion

$$
\sqrt{\mathrm{T}}\left(\widehat{\theta}-\theta_{0}\right)=\mathrm{U}_{\mathrm{T}}(\theta)+\mathrm{T}^{-1} \xi_{\mathrm{T}}
$$

where $\xi_{\mathrm{T}}$ satisfies $\mathbf{P}_{\theta_{0}}\left(\left|\xi_{\mathrm{T}}\right|>\rho_{\mathrm{T}} \sqrt{\mathrm{T}}\right)=\mathrm{o}\left(\mathrm{T}^{-1 / 2}\right)$ for some sequence $\rho_{\mathrm{T}} \rightarrow 0, \rho_{\mathrm{T}} \sqrt{\mathrm{T}} \rightarrow \infty$ as $\mathrm{T} \rightarrow \infty$.

\section{Proof.}

From the equation $\mathrm{L}_{\mathrm{T}}^{(1)}(\hat{\theta})=0$, we have

$0=\sqrt{T} \mathrm{~L}_{\mathrm{T}}^{(1)}\left(\theta_{0}\right)+\frac{1}{\sqrt{\mathrm{T}}} \mathrm{Z}_{2}\left(\theta_{0}\right) \mathrm{V}_{\mathrm{T}}+\mathbf{E}_{\theta_{0}} \mathrm{~L}_{\mathrm{T}}^{(2)}\left(\theta_{0}\right) \mathrm{V}_{\mathrm{T}}+\frac{1}{2 \sqrt{\mathrm{T}}} \mathrm{L}_{\mathrm{T}}^{(3)}\left(\theta_{0}\right) \mathrm{V}_{\mathrm{T}}^{2}+\frac{1}{6 \mathrm{~T}} \mathrm{~L}_{\mathrm{T}}^{(4)}(\widetilde{\theta}) \mathrm{V}_{\mathrm{T}}^{3}$

where $\left|\widetilde{\theta}-\theta_{0}\right| \leq\left|\widehat{\theta}-\theta_{0}\right|$.

We rewrite (4.31) as 


$$
\begin{aligned}
\mathrm{V}_{\mathrm{T}}=-\frac{\sqrt{\mathrm{T}} \mathrm{L}_{\mathrm{T}}^{(1)}\left(\theta_{0}\right)}{\mathbf{E}_{\theta_{0}} \mathrm{~L}_{\mathrm{T}}^{(2)}\left(\theta_{0}\right)}-\frac{1}{\mathbf{E}_{\theta_{0}} \mathrm{~L}_{\mathrm{T}}^{(2)}\left(\theta_{0}\right) \sqrt{\mathrm{T}}} \mathrm{Z}_{2}\left(\theta_{0}\right) \mathrm{V}_{\mathrm{T}}-\frac{\mathrm{L}_{\mathrm{T}}^{(3)}\left(\theta_{0}\right)}{2 \mathbf{E}_{\theta_{0}} \mathrm{~L}_{\mathrm{T}}^{(2)}\left(\theta_{0}\right) \sqrt{\mathrm{T}}} \mathrm{V}_{\mathrm{T}}^{2} \\
-\frac{\mathrm{L}_{\mathrm{T}}^{(4)}(\tilde{\theta})}{6 \mathbf{E}_{\theta_{0}} \mathrm{~L}_{\mathrm{T}}^{(2)}\left(\theta_{0}\right) \mathrm{T}} \mathrm{V}_{\mathrm{T}}^{3}
\end{aligned}
$$

Noting (3.4), (4.27) and Lemma 4.4 with $0<\alpha<1 / 10$, we can write (4.32) as

$$
\mathrm{V}_{\mathrm{T}}=-\frac{\sqrt{\mathrm{T}} \mathrm{L}_{\mathrm{T}}^{(1)}\left(\theta_{0}\right)}{\mathbf{E}_{\theta_{0}} \mathrm{~L}_{\mathrm{T}}^{(2)}\left(\theta_{0}\right)}+\frac{1}{\sqrt{\mathrm{T}}} \tilde{\xi}_{\mathrm{T}}
$$

where $\mathbf{P}_{\theta_{0}}\left(\left|\tilde{\xi}_{\mathrm{T}}\right|>\mathrm{d}_{8} \mathrm{~T}^{2 \alpha}\right)=\mathrm{o}\left(\mathrm{T}^{-1 / 2}\right)$ for some $\mathrm{d}_{8}>0$.

Substituting (4.33) for the right-hand side of (4.32) and noting that

$$
\mathbf{E}_{\theta_{0}} \mathrm{~L}_{\mathrm{T}}^{(1)}\left(\theta_{0}\right)=\mathrm{o}\left(\mathrm{T}^{-1}\right) \text { and } \mathbf{E}_{\theta_{0}} \mathrm{~L}_{\mathrm{T}}^{(2)}\left(\theta_{0}\right)=\mathrm{L}^{(2)}\left(\theta_{0}\right)+\mathrm{o}\left(\mathrm{T}^{-1}\right) \text { by Lemma 4.3(i) }
$$

we have

$V_{T}=-\frac{Z_{1}\left(\theta_{0}\right)}{L^{(2)}\left(\theta_{0}\right)}+\frac{1}{\sqrt{T}} \frac{Z_{1}\left(\theta_{0}\right)}{L^{(2)}\left(\theta_{0}\right)} \frac{Z_{2}\left(\theta_{0}\right)}{L^{(2)}\left(\theta_{0}\right)}-\frac{1}{2 \sqrt{T}} \frac{L^{(3)}\left(\theta_{0}\right)}{L^{(2)}\left(\theta_{0}\right)}\left(\frac{Z_{1}\left(\theta_{0}\right)}{L^{(2)}\left(\theta_{0}\right)}\right)^{2}+\frac{1}{T} \xi_{T}$,

where $\mathbf{P}_{\theta_{0}}\left(\left|\xi_{\mathrm{T}}\right|>\mathrm{d}_{9} \mathrm{~T}^{3 \alpha}\right)=\mathrm{o}\left(\mathrm{T}^{-1 / 2}\right)$, for some $\mathrm{d}_{9}>0$.

Since $0<\alpha<1 / 10$, we have the desired result.

\section{Proof of (2) in Theorem 3.1.}

By Lemma 4.6 the Edgeworth expansion for $\sqrt{\mathrm{T}}\left(\hat{\theta}-\theta_{0}\right)$ (up to order $\mathrm{T}^{-1 / 2}$ ) is equal to that of $U_{T}\left(\theta_{0}\right)$. Thus we have to derive the Edgeworth expansion for $U_{T}\left(\theta_{0}\right)$. Since $U_{T}\left(\theta_{0}\right)$ is a smooth function of $Z_{1}\left(\theta_{0}\right)$ and $Z_{2}\left(\theta_{0}\right)$ this expansion follows from the expansion of the vector $\left(\mathrm{Z}_{1}\left(\theta_{0}\right), \mathrm{Z}_{2}\left(\theta_{0}\right)\right)$ by the well-known Transformation-Lemma (cf. Bhattacharya and Ghosh (1978)). 


\section{References}

Bentkus, R. and Rudzkis, R. A. (1982). On the distribution of some statistical estimates of spectral density. Theory Prob. and Appl. 27795 - 814.

Bhattacharya, R. N. and Ranga Rao, R. (1976). Normal approximation and asymptotic expansions. Wiley, New York.

Bhattacharya, R. N. and Ghosh, J. K. (1978). On the validity of the formal Edgeworth expansion. Ann. Statist. 6434 - 451.

Bose, A. (1988). Higher order approximations for autocovariances from linear processes with applications. Statistics 19259 - 269.

Brillinger, D. R. (1981). Time series, data analysis and theory. Mc Graw-Hill, Inc., New York.

Dahlhaus, R. (1983). Spectral analysis with tapered data. J. Time Ser. Anal. 4, 163 - 175.

Dzhaparidze, K. O. and Yaglom, A. M. (1983). Spectrum parameter estimation in time series analysis. In: Krishnaiah, P. R.. Developments in Statistics. Vol. 4 (Academic Press, New York).

Götze, F. and Hipp, C. (1983). Asymptotic expansions for sums of weakly dependent random vectors. Zeit. Wahr. verw. Gebiete 64211 - 239.

Taniguchi, M. (1991). Higher order asymptotic theory for time series analysis.

Springer, New York. 